\title{
Zur Trennung des Kalks von der Magnesia.
}

\author{
Von
}

\section{Demselben.}

Bereits in der 1857 erschienenen vierten Auflage meiner Anleitung zur quantitativen Analyse habe ich mit grösster Bestimmtheit darauf aufmerlrsam gemacht, dass bei Trennung von Kalk und Magnesia durch oxalsaures Ammon bei Gegenwart von Chlorammonium in Flüssigkeiten, welche relativ viel Magnesia enthalten, bei bloss einmaliger Fällung stets nicht ganz richtige Resultate erhalten würden, weil unter diesen Umständen mit dem oxalsauren Kalke stets etwas Magnesia als oxalsaure Magnesia oder oxalsaure Ammonmagnesia niederfalle.

Ich empfahl daher den erstgefällten oxalsauren Kalk nach einmaligem Auswaschen nochmals in Salzsäure zu lösen, die Lösung durch Ammon unter Zusatz von etwas oxalsaurem Ammon wiederum zu fällen, erst diesen zweiten Niederschlag als zur Gewichtsbestimmung des Kalkes geeignet $\mathrm{zn}$ betrachten, in den vereinigten Filtraten aber, nach Entfernung der Ammonsalze, die Magnesia zu bestimmen.

Diese meine Trennungsmethode scheint im Ganzen bei den Chemikern, welche sich vorzugsweise mit analytischer Chemie beschäftigen, nicht viel Anklang gefunden zu baben, nicht weil man bei eingehender Prüfung der Sache meinen Erfahrungen widersprechende Resultate gefunden hätte, sondern offenbar weil man die Trennung durch einmalige Fällang als eine durch langjährige Uebnng sanctionirte betrachtete und sich ausserdem nicht entschliessen konnte, an Stelle der kürzeren und bequemeren die langwierigere Methode zu setzen. - In der That hat weder H. Rose*), noch Rivot**) p. 48.

*) H. Rose, Traité complet de Chimie analytique, Anal. quant. (1862)

**) Handbuch der analytischen Mineralchemie von Rivot, deutsch von Remelé, 1866, Bd. II. S. 416. 
noch Finkener*) sich bewogen gefunden, die doppelte Fällung zu empfehlen.

Obgleich ich nun in meiner Anleit. zur quant. An. 5. Autl. Ṡ. 955 schon eine Reihe von Versuchen mittheilte, aus denen sich die

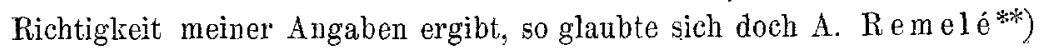
zu dem folgenden Ausspruche berechtigt:

„Es scheint, dass dieser ganzen Sache (Einwirlkung des lösenden Einflusses der Magnesiasalzlösungen auf oxalsauren Kalk bei Abwesenheit grösserer Mengen von oxalsaurem Ammon und Niederfallen kleinerer Magnesiamengen mit dem oxalsauren Kalk aus an Magnesia reichen Iösungen bei nur einmaliger Fällung) zu grosse Wichtigkeit beigelegt worden ist. Man hat für die Scheidung von Kalk und Magnesia neue Schwierigkeiten geschaffen, die theoretisch begründet sein mögen, welche aber vielleicht den Arbeiter mit keiner grösseren Genauigkeit belohnen. Seit geraumer Zeit haben sich die vorsichtigsten und geschicktesten Chemiker der von Herrn Rivot wie auch anderwärts beschriebenen Verfahrungsweise für die Trennung jener Basen (nämlich der einmaligen Fällung mit uberschüssigem oxalsaurem Ammon bei Salmiakzusatz) mit dem besten Erfolge bedient; die Methode ist durch die Erfahrung vollkommen bewährt."

Da es in der Wissenschaf́t wie im Leben żweckmässig ist einmal angeregte Fragen $z u$ sicherem Abschlusse zu bringen, so theile ich im Folgenden zwei mit der grössten Sorgfalt und in grossem Maassstabe durchgeführte Versuche mit, welche die Sache vollkommen klar zu stellen geeignet sein werden.

Das bekannte im Brohlthal gelegene Mineralwasser des Heilbrunnens, mit dessen Ánalyse ich eben beschäftigt bin, bot zu den Versuchen eine recht gute Gelegenheit.

8240,6 Grm. desselben wurden - nach Abscheidung der Kieselsäure und des Eisens und der höchst geringen Mengen von Phosphoräure und Thonerde - bei Anwesenbeit ciner reichlich genügenden Menge von Chlorammonium mit Ammon versetzt und der vollkommen lslaren Lösung reines oxalsaures Ammon in entsprechendem Ueberschusse zugefügt. Nach 15 Stunden Stehens bei etwa $20^{\circ} \mathrm{C}$. wurde der Nie-

*) H. Rose, Handbuch der analytischen Chemie, sechste Auflage, vollendet von R. Finkener, Bd. II. S. 44.

**) A. a. O. S. 419 . 
derschlag abfiltrirt, vollkommen ansgewaschen, wieder in Salzsäure gelöst, die Lösung. mit Ammon und etwas oxalsaurem Ammon versetzt, der Niederschlag nach dem Absitzen neuerdings abfiltrirt, ausgewaschen, getrocknet, durch regelrechtes Erhitzen in reinen kohlensauren Kalk übergeführt und dieser gewogen. Man erhielt 3,0252 Grm. -

Um nun zu entscheiden, ob und wieviel Magnesia bei der ersten Fällung mit dem oxalsauren Kalk niedergeschlagen wurde, behandelte ich die von der ersten Fallung and die von der zweiten Fällung des oxalsauren Kalks abfiltrirte Flüssigkeit nicht zusammen, wie ich diess gewohnt bin, sondern getrennt. Enthielt der erste Kalkniederschlag Magnesia, so musste sich diese in der vom zweiten abfiltrirten Lösung finden.

Jedes der beiden Filtrate wurde in einer grossen Platinschale zur Trockne gebracht. Man entfernte dann den grössten Theil der Ammonsalze durch Glühen und bestimmte im Rückstande die Magnesia durch Fällung als phosphorsaure Ammonmagnesia etc.

Das erste Filtrat lieferte 11,2204 Grm. pyrophosphorsaure Magnesia, das zweite 0,0359 Grm. Als die geglühten Rückstände in Salzsäure gelöst, die Lösungen mit Schwefelsäure versetzt und mit. viel starkem Alkohol vermischt wurden, blieben sie auch bei langem Stehen klar; sie waren somit kalkfrei.

$6278,1 \mathrm{Grm}$. desselben Wassers lieferten bei gleicher Behandlung 2,3038 Grm. kohlensauren Kalk, 8,5350 Grm. pyrophosphorsaure Magnesia aus dem ersten Filtrat und $0,0410 \mathrm{Grm}$. aus dem zweiten Filtrate.

Vergleichen wir nun die Resultate, wie sich solche bei einmaliger Fãllung des Kalks würden ergeben haben, mit den richtigen, welche bei zweimaliger Fälluing erhalten wurden.

0,0359 Grm. pyrophosphorsaure Magnesia entsprechen 0,0129 Grm. reiner Magnesia; als solche würde diese in dem gewogenen kohlensauren Kalk enthalten gewesen sein; es wären somit gefunden worden $3,0252+0,0129=3,0381$ Grm. kohlensaurer Kalk oder 0,368674 pm.

Als kohlensaure Magnesia aber hätte man erhalten die 11;2204 Grm. entsprechende Menge, nämlich 8,4911 Grm. oder 1,030398 pm.

Bei der zweiten 'Analyse wäre gefunden worden kohlensaurer Kalk: 2,3038+0,0148 (Menge der Magnesia, welche 0,041 pyrophosphorsaurer Magnesia entspricht) $=2,3186$ oder $0,369315 \mathrm{pm}$. 
und als kohlensaure Magnesia: 6,4589 Grm. oder 1,028798 pm. d. h. die den 8,5350 Grm. pyrophosphorsaurer Magnesia entsprechende Menge.

Die Differenzen ergeben sich aus folgender Uebersicht:

Ungenaue Resultate bei ejnmaliger Kalkfällung:

$\begin{array}{lccc} & \text { 1. Best. } & \text { 2. Best. } & \text { Mittel. } \\ \text { Kohlensaurer Kalk } & 0,368674 & 0,369315 & 0,368994 \\ \text { Kohlensaure Magnesia } & \mathbf{1 , 0 3 0 3 9 8} & \mathbf{1 , 0 2 8 7 9 8} & \mathbf{1 , 0 2 9 5 9 8}\end{array}$

Genaue Resultate bei doppelter Kalkfällung:

$\begin{array}{llll}\text { Kohlensaurer Kalk } & 0,367109 & 0,366958 & 0,367033 \\ \text { Kohlensaure Magnesia } & 1,033699 & 1,033736 & 1,033718\end{array}$

Differenzen der angenauen Bestimmungen von den geriauen im Mittel: Kohlensaurer Kalk $+0,001961$ Kohlensaure Magnesia -0,004120

Die Schlussfolge ist nun einfach die: wem es bei Analysen auf Differenzen der angegebenen Art nicht ankommt, der kann es bei einmaliger Kalkfällung bewenden lassen, wer dagegen solche Fehler vermeiden will, der muss sich zur zweimaligen Fällung entschliessen.

\section{Chemisch-analytische Beiträge. Yon}

\section{D. Braun.}

Dritte Abhandlung.

1. Zur Kenntniss der sog. salpetrigsauren KobaltoxydKaliverbindungen und verwandter Producte.

Die Frage, ob sich die salpetrige Säure mit Kobaltoxydul vereinigen könne, ist noch nicht entscheidend gelöst worden. J. Lang spricht bei Gelegenheit der Beschreibung verschiedener salpetrigsaurer 